\title{
Clostridioides difficile: un patógeno peculiar
}

Flora Cruz-López

flora.cruz@live.com

Alejandra Sánchez-Muñoz

alesanchez123430@gmail.com

Adrián Martínez-Meléndez

adrian.mtz.fcq@gmail.com

Facultad de Ciencias Químicas, Universidad Autónoma de Nuevo León. Pedro de Alba S/N, Ciudad Universitaria, CP 66450, San Nicolás de los Garza, Nuevo León, México.

\section{RESUMEN}

La infección por la bacteria Clostridioides difficile (ICD) se desarrolla principalmente en pacientes hospitalizados y tratados con antibióticos, particularmente en adultos mayores o pacientes inmunocomprometidos. La enfermedad puede resultar en la muerte y cursar con múltiples recaídas. La principal causa de la ICD es la administración de antibióticos, ya que causan alteración de la microbiota intestinal, lo cual permite el desarrollo de $C$. difficile. Sin embargo, el tratamiento de primera línea para la infección por $C$. difficile se basa en antibióticos. Recientemente, el trasplante de microbiota fecal también se ha descrito como una alternativa, ya que parece ser seguro y sin efectos adversos.

Palabras clave: clostridioides difficile; infecciones hospitalarias; diarrea infecciosa. 


\title{
Clostridioides difficile: a peculiar pathogen
}

\begin{abstract}
Infection with Clostridioides difficile (CDI) develops mainly in hospitalized patients who are treated with antibiotics, particularly in older adults or immunocompromised patients. This disease can result in death and lead to multiple relapses. The main cause of CDI is the administration of antibiotics, since they cause alteration of the intestinal microbiota, which allows the development of $C$. difficile. However, first-line treatment for $C$. difficile infection is based on antibiotics. Recently, fecal microbiota transplantation has also been described as an alternative, since it appears to be safe and without adverse effects.
\end{abstract}

Keywords: Clostridioides difficile; hospital infections; infectious diarrhea.

Artículo recibido: 30 noviembre. 2021 Aceptado para publicación: 29 diciembre 2021

Correspondencia: flora.cruz@live.com Conflictos de Interés: Ninguna que declarar 


\section{INTRODUCCIÓN}

La infección por la bacteria Clostridioides difficile (ICD) ha cobrado importancia en los últimos años, ya que se desarrolla principalmente en pacientes hospitalizados y tratados con antibióticos, particularmente en adultos mayores o pacientes inmunocomprometidos. La enfermedad posee diversas presentaciones, que pueden ir desde la infección sin síntomas, episodios de diarrea acuosa, hasta una inflamación grave del intestino grueso que puede resultar en la muerte; además, la infección puede cursar con múltiples recaídas, cuya probabilidad aumenta de manera consecutiva. Según información del CDC (Centers for Disease Control and Prevention) se estima que $C$. difficile causa 500,000 infecciones al año en los Estados Unidos, y que uno de cada once pacientes mayores de 65 años, muere por ICD.

\section{Clostridiodides difficile: un patógeno emergente}

C. difficile es la causa infecciosa más común de diarrea en hospitales. C. difficile es un patógeno que se puede considerar de reciente descripción, ya que fue en 1978 cuando se asoció por primera vez a cuadros de colitis, particularmente en pacientes que recibían tratamiento con clindamicina, un antibiótico de reciente desarrollo en ese tiempo (Elliott, Androga, Knight, \& Riley, 2017). Este microorganismo es capaz de producir de esporas; esto es, produce una estructura resistente alrededor del material genético de la bacteria cuando las condiciones ambientales no son favorables, manteniéndola en un estado de reposo en el cual puede soportar condiciones adversas y mantenerse viable durante periodos prolongados de tiempo (Shen, 2020).

C. difficile vive de manera natural en el tracto intestinal de ciertos animales y puede colonizar el de los seres humanos, principalmente cuando los individuos se encuentran hospitalizados. Además, C. difficile coloniza del $15 \%$ al $70 \%$ de los infantes y aproximadamente el 5\% de adultos sanos (M. J. T. Crobach et al., 2018). En el entorno hospitalario, las esporas se pueden encontrar fácilmente debido a la propagación en superficies como mesas de noche, camas, grifos, manijas o también por el contacto con los profesionales de salud como médicos, enfermeras, camilleros, entre otras superficies (Figura 1). Las esporas de C. difficile pueden persistir en el ambiente y son resistentes a los desinfectantes de base en alcohol y de otros tipos (Barbut, 2015; Czepiel et al., 2019). 


\section{¿Cómo causa enfermedad $C$. difficile?}

La ICD se asocia principalmente a la disbiosis del tracto gastrointestinal; es decir, a un desequilibrio en las comunidades microbianas que viven normalmente en el tracto intestinal y que son necesarias para muchos procesos metabólicos en nuestro cuerpo (Theriot \& Young, 2015). A estas comunidades microbianas también se les conoce como microbiota intestinal, y la disminución o la alteración de estas comunidades puede traer consecuencias a la salud; tal es el caso de la ICD. Cuando la microbiota se ve disminuida o alterada, las esporas de $C$. difficile pueden activarse y dar lugar a la bacteria metabólicamente activa y capaz de producir enfermedad. Lo anterior sucede en el intestino grueso, ya que este segmento de nuestro tracto gastrointestinal es anaerobio (libre de oxígeno) y es el ambiente ideal para que $C$. difficile pueda colonizar y crecer fácilmente (Czepiel et al., 2019; Smits, Lyras, Lacy, Wilcox, \& Kuijper, 2016).

Figura 1. Proceso de diseminación e infección por C. difficile.

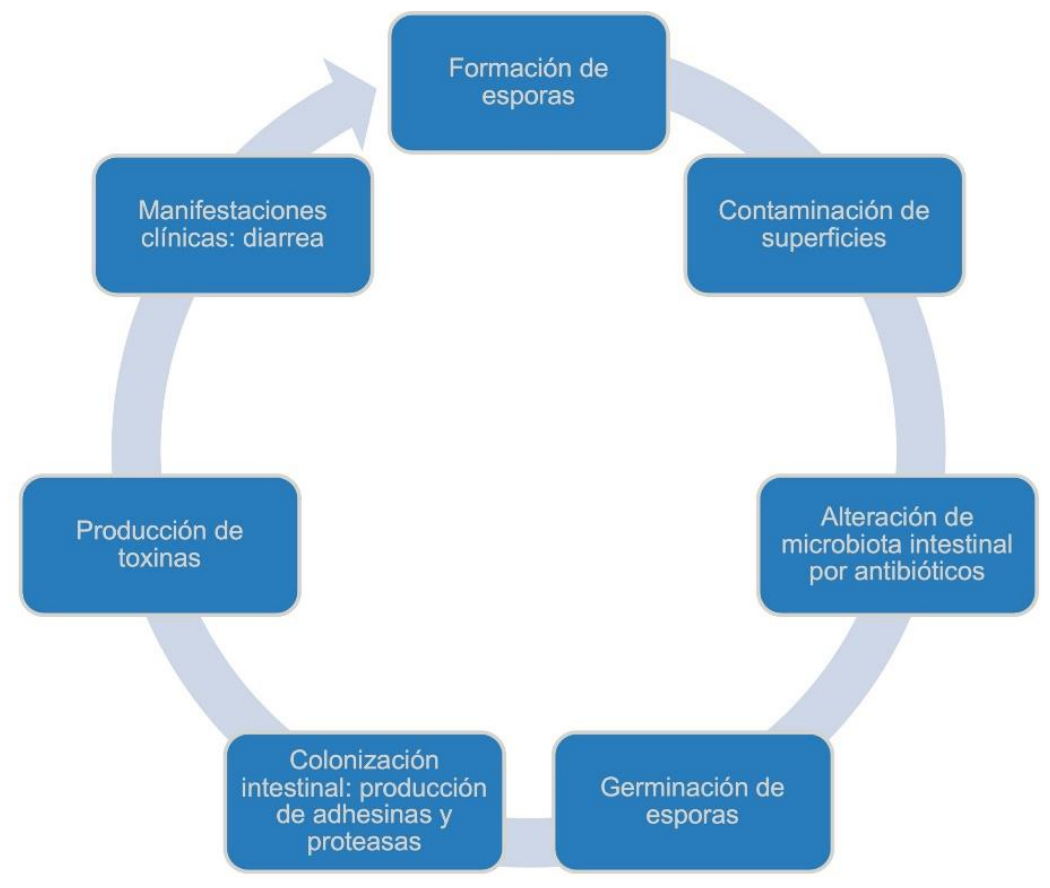

La causa principal de disbiosis en los pacientes hospitalizados se debe a la administración de antibióticos (Smits et al., 2016). Comúnmente, los antibióticos nos ayudan a combatir infecciones bacterianas; sin embargo, al consumir antibióticos no solo las bacterias que están causando alguna infección se ven afectadas, sino que también afectan a los microorganismos de la microbiota intestinal (Abt, McKenney, \& Pamer, 2016). Por lo 
tanto, las alteraciones provocadas por los antibióticos permiten el desarrollo de $C$. difficile. Además, existen pacientes que presentan mayor riesgo de desarrollar ICD como los pacientes mayores de 65 años, pacientes con inmunosupresión, cáncer o pacientes que han sido trasplantados (Mullish \& Williams, 2018).

Hay que aclarar que la sola presencia de $C$. difficile no provoca enfermedad o sintomatología, si no que esta bacteria posee mecanismos llamados factores de virulencia, que son sustancias que provocan los daños en el individuo y por lo tanto la sintomatología (Figura 2). Dentro de los factores de virulencia que produce $C$. difficile, se encuentra la producción de toxinas. $C$. difficle produce principalmente dos toxinas conocidas como toxina A (TcdA) y toxina B (TcdB), las cuales causan la muerte y el desprendimiento de las células epiteliales del intestino e inflamación en el sitio de la infección (Chandrasekaran \& Lacy, 2017).

Figura 2. Proceso de germinación y daños causados por C. difficile

\section{Producción de toxinas}

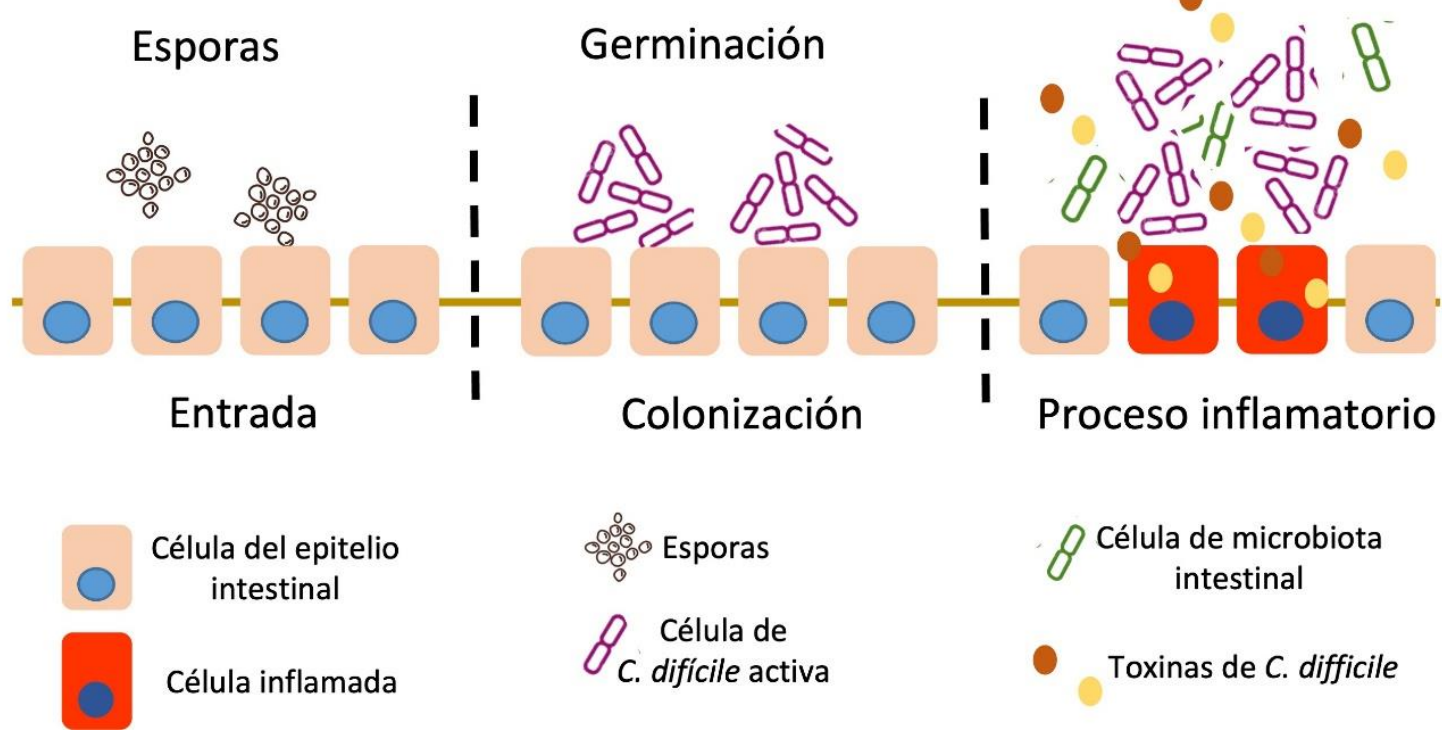

\section{Síntomas y diagnóstico}

La ICD puede manifestarse de diferentes maneras para el individuo infectado por $C$. difficile, una de ellas es que los pacientes la padezcan de forma asintomática, lo cual es más frecuente en niños que en adultos. Por otro lado, la ICD puede presentarse como diarrea leve o moderada, colitis pseudomembranosa (por la presencia de placas elevadas y amarillas en el colon), e íleo y megacolon tóxico, las cuales pueden ser mortales. La 
diarrea por $C$. difficile se manifiesta como heces liquidas o acuosas, acompañada con frecuencia de dolores abdominales, nauseas y fiebre (Hookman \& Barkin, 2009). La ICD se puede diagnosticar por medio del análisis de las heces del paciente, al buscar las toxinas libres por medio de inmunoensayos o bien por la detección molecular del ADN de la bacteria (M. J. Crobach et al., 2016). También se pueden observar las pseudomembranas formadas por medio de endoscopia, o bien después de una colectomía (la extirpación del colon o parte de este) o una autopsia en el caso de fallecimiento (Hookman \& Barkin, 2009). En ocasiones, la ICD se presenta nuevamente después de haberse aliviado por completo los síntomas de una primera infección; lo cual es conocido como recurrencia y puede deberse a que $C$. difficile no fue eliminado por completo a pesar del tratamiento, o bien a una nueva infección (Chilton, Pickering, \& Freeman, 2018). Esto ocurre generalmente dentro de las primeras 2-12 semanas tras la resolución de los síntomas; sin embargo, el hecho de haber presentado una recurrencia es en sí mismo un factor de riesgo para continuar presentando recaídas (McDonald et al., 2018; Song \& Kim, 2019).

\section{Tratamiento}

La primera indicación en los pacientes es retirar todo aquel antibiótico que no sea necesario administrar; en muchas ocasiones, la ICD cesa si se le deja de administrar antibióticos a los pacientes al restaurarse la microbiota intestinal (Ooijevaar et al., 2018). Si embargo, el tratamiento de primera línea para la infección por C. difficile precisamente se basa en antibióticos, específicamente metronidazol y vancomicina (van Prehn et al., 2021). El régimen por utilizar depende de si se trata de una infección inicial o infecciones recurrentes y también dependiendo cuantas veces se haya presentado. A pesar de ello, se consideran los mismos antibióticos, pero en diferentes dosis y vías de administración (van Prehn et al., 2021). Sin embargo, el tratamiento tradicional ha tenido dificultades para resolver las infecciones, por diferentes razones, primeramente, el fallo en el tratamiento con metronidazol se ha incrementado, por lo que se opta por tratar a los pacientes con vancomicina inicialmente (Manthey, Eckmann, \& Fuhrmann, 2017). En años recientes se ha introducido la fidaxomicina, un antibiótico administrado por vía oral que no afecta considerablemente a la microbiota y que se ha demostrado su efectividad (Zhanel, Walkty, \& Karlowsky, 2015). Actualmente se encuentra en las guías clínicas de algunos países industrializados; desafortunadamente, este antibiótico no se encuentra disponible en México (McDonald et al., 2018). 
El trasplante de microbiota fecal es un tratamiento que continúa en estudio, pero que promete una solución a la infección recurrente. Consisten en la administración de un preparado de heces de donadores sanos, y se administra en forma de enemas o con tubos nasogástricos o duodenales para permitir la restauración de la microbiota intestinal y la resolución de los síntomas incluso en horas. El transplante de microbiota fecal es prometedor, ya que parece ser seguro y sin efectos adversos (McDonald et al., 2018; Vindigni \& Surawicz, 2017).

\section{CONCLUSIÓN}

La infección por $C$. difficile es un problema de suma importancia en hospitales de todo el mundo y que pone en riesgo la vida del paciente. Este microorganismo es de reciente descripción y por lo tanto, aún hay mucho que conocer acerca de su proceso infeccioso. Sin embargo, la información se encuentra en constante evolución, y muchas interrogantes serán resueltas, lo que contribuirá a obtener diagnósticos más certeros y mejores terapias farmacológicas, con todos aquellos nuevos tratamientos que siguen en fases de aprobación y nuevas terapias como el trasplante fecal.

\section{REFERENCIAS BIBLIOGRAFICAS}

Abt, M. C., McKenney, P. T., \& Pamer, E. G. (2016). Clostridium difficile colitis: pathogenesis and host defence. Nat Rev Microbiol, 14(10), 609-620. doi:10.1038/nrmicro.2016.108. https://www.ncbi.nlm.nih.gov/pubmed/27573580.

Barbut, F. (2015). How to eradicate Clostridium difficile from the environment. J Hosp Infect, $\quad$ 89(4), 287-295. doi:10.1016/j.jhin.2014.12.007. https://www.ncbi.nlm.nih.gov/pubmed/25638358.

Chandrasekaran, R., \& Lacy, D. B. (2017). The role of toxins in Clostridium difficile infection. FEMS Microbiol Rev, 41(6), 723-750. doi:10.1093/femsre/fux048. https://www.ncbi.nlm.nih.gov/pubmed/29048477.

Chilton, C. H., Pickering, D. S., \& Freeman, J. (2018). Microbiologic factors affecting Clostridium difficile recurrence. Clin Microbiol Infect, 24(5), 476-482. doi:10.1016/j.cmi.2017.11.017. https://www.ncbi.nlm.nih.gov/pubmed/29208562.

Crobach, M. J., Planche, T., Eckert, C., Barbut, F., Terveer, E. M., Dekkers, O. M., . . Kuijper, E. J. (2016). European Society of Clinical Microbiology and Infectious Diseases: update of the diagnostic guidance document for Clostridium difficile 
infection. Clin Microbiol Infect, 22 Suppl 4, S63-81. doi:10.1016/j.cmi.2016.03.010. https://www.ncbi.nlm.nih.gov/pubmed/27460910.

Crobach, M. J. T., Vernon, J. J., Loo, V. G., Kong, L. Y., Pechine, S., Wilcox, M. H., \& Kuijper, E. J. (2018). Understanding Clostridium difficile Colonization. Clin Microbiol Rev, 31(2). doi:10.1128/CMR.00021-17. https://www.ncbi.nlm.nih.gov/pubmed/29540433.

Czepiel, J., Drozdz, M., Pituch, H., Kuijper, E. J., Perucki, W., Mielimonka, A., . . . Biesiada, G. (2019). Clostridium difficile infection: review. Eur J Clin Microbiol Infect Dis, 38(7), 1211-1221. doi:10.1007/s10096-019-03539-6. https://www.ncbi.nlm.nih.gov/pubmed/30945014.

Elliott, B., Androga, G. O., Knight, D. R., \& Riley, T. V. (2017). Clostridium difficile infection: Evolution, phylogeny and molecular epidemiology. Infect Genet Evol, 49, $1-11$. doi:10.1016/j.meegid.2016.12.018. https://www.ncbi.nlm.nih.gov/pubmed/28012982.

Hookman, P., \& Barkin, J. S. (2009). Clostridium difficile associated infection, diarrhea and colitis. World J Gastroenterol, 15(13), 1554-1580. doi:10.3748/wjg.15.1554. https://www.ncbi.nlm.nih.gov/pubmed/19340897.

Manthey, C. F., Eckmann, L., \& Fuhrmann, V. (2017). Therapy for Clostridium difficile infection - any news beyond Metronidazole and Vancomycin? Expert Rev Clin Pharmacol, 10(11), 1239-1250. doi:10.1080/17512433.2017.1362978. https://www.ncbi.nlm.nih.gov/pubmed/28766951.

McDonald, L. C., Gerding, D. N., Johnson, S., Bakken, J. S., Carroll, K. C., Coffin, S. E., . . . Wilcox, M. H. (2018). Clinical Practice Guidelines for Clostridium difficile Infection in Adults and Children: 2017 Update by the Infectious Diseases Society of America (IDSA) and Society for Healthcare Epidemiology of America (SHEA). Clin Infect Dis, 66(7), e1-e48. doi:10.1093/cid/cix1085. https://www.ncbi.nlm.nih.gov/pubmed/29462280.

Mullish, B. H., \& Williams, H. R. (2018). Clostridium difficile infection and antibioticassociated diarrhoea. Clin Med (Lond), 18(3), 237-241. doi:10.7861/clinmedicine.18-3-237. https://www.ncbi.nlm.nih.gov/pubmed/29858434. 
Ooijevaar, R. E., van Beurden, Y. H., Terveer, E. M., Goorhuis, A., Bauer, M. P., Keller, J. J., . . . Kuijper, E. J. (2018). Update of treatment algorithms for Clostridium difficile infection. Clin Microbiol Infect, 24(5), 452-462. doi:10.1016/j.cmi.2017.12.022. https://www.ncbi.nlm.nih.gov/pubmed/29309934.

Shen, A. (2020). Clostridioides difficile Spore Formation and Germination: New Insights and Opportunities for Intervention. Annu Rev Microbiol, 74, 545-566. doi:10.1146/annurev-micro-011320-011321.

https://www.ncbi.nlm.nih.gov/pubmed/32905755.

Smits, W. K., Lyras, D., Lacy, D. B., Wilcox, M. H., \& Kuijper, E. J. (2016). Clostridium difficile infection. Nat Rev Dis Primers, 2, 16020. doi:10.1038/nrdp.2016.20. https://www.ncbi.nlm.nih.gov/pubmed/27158839.

Song, J. H., \& Kim, Y. S. (2019). Recurrent Clostridium difficile Infection: Risk Factors, Treatment, and Prevention. Gut Liver, 13(1), 16-24. doi:10.5009/gnl18071. https://www.ncbi.nlm.nih.gov/pubmed/30400734.

Theriot, C. M., \& Young, V. B. (2015). Interactions Between the Gastrointestinal Microbiome and Clostridium difficile. Annu Rev Microbiol, 69, 445-461. doi:10.1146/annurev-micro-091014-104115.

https://www.ncbi.nlm.nih.gov/pubmed/26488281.

van Prehn, J., Reigadas, E., Vogelzang, E. H., Bouza, E., Hristea, A., Guery, B., . . . Guideline Committee of the European Study Group on Clostridioides, d. (2021). European Society of Clinical Microbiology and Infectious Diseases: 2021 update on the treatment guidance document for Clostridioides difficile infection in adults. Clin Microbiol Infect, 27 Suppl 2, S1-S21. doi:10.1016/j.cmi.2021.09.038. https://www.ncbi.nlm.nih.gov/pubmed/34678515.

Vindigni, S. M., \& Surawicz, C. M. (2017). Fecal Microbiota Transplantation. Gastroenterol Clin North Am, 46(1), 171-185. doi:10.1016/j.gtc.2016.09.012. https://www.ncbi.nlm.nih.gov/pubmed/28164849.

Zhanel, G. G., Walkty, A. J., \& Karlowsky, J. A. (2015). Fidaxomicin: A novel agent for the treatment of Clostridium difficile infection. Can J Infect Dis Med Microbiol, 26(6), 305-312. doi:10.1155/2015/934594. https://www.ncbi.nlm.nih.gov/pubmed/26744587. 